borate buffer ( $\mathrm{pH}$ 7.7) and placed in an autosampler HPLC system (Merck-Hitachi, Darmstad, Germany) that automatically performs the fluorenyl methoxycarbonyl (FMOC) derivatization by adding $250 \mu$ l of a $15 \mathrm{mM}$ solution of 9-fluoromethoxycarbonylchloride (FMOC-Cl; Sigma-Aldrich, Milano, Italy) in acetone and, after $45 \mathrm{~s}$, removing the excess reagent by two pentane extractions $(900 \mu \mathrm{l})$. HPLC-grade reagents were purchased from Romil (Cambridge, UK) rpHPLC analyses of the FMOC-derivatized compounds were performed under the following conditions: A column RP-8 Superspher 60 LiChroCart was used; eluents were $\mathrm{H}_{2} \mathrm{O} / \mathrm{CH}_{3} \mathrm{CN}(80: 20)$ and 40 $\mathrm{mM} \mathrm{CH}{ }_{3} \mathrm{COONa}\left(\mathrm{pH}\right.$ 4.6) (A); $\mathrm{H}_{2} \mathrm{O} / \mathrm{CH}_{3} \mathrm{CN}(20: 80)$ and $10 \mathrm{mM} \mathrm{CH}_{3} \mathrm{COONa}(\mathrm{pH} 7.0)(\mathrm{B})$; and $\mathrm{CH}_{3} \mathrm{CN}$ (C). Gradient: 0 min, $100 \%$ A; 30 min, $50 \%$ A, 50\% B; 40 min, $100 \%$ C; 45 min, 100\% C; 50 min, 100\% A. Flow was at $1.25 \mathrm{ml} / \mathrm{min}$ at a temperature of $45^{\circ} \mathrm{C}$; ultraviolet monitoring was used (wavelength, 263 $\mathrm{nm})$. The concentration of thiols in the samples was determined by comparison with calibrated amounts of standard solutions of cysteine, GSH, GSSG, and the mixed disulfide between cysteine and glutathione (Cys-SG). The identity of individual peaks in the chromatograms was further confirmed by time-of-flight matrix-assisted laser desorption ionization mass spectrometry (4) [T. Tanaka et al., Rapid Comm. Mass Spectrom. 2,151 (1988)].

25. For pulse and chase analysis, injected oocytes were cultured overnight at $20^{\circ} \mathrm{C}$ in modified Barths' saline, washed, and resuspended in modified Barths' saline (5 $\mu$ l per oocyte) containing [ ${ }^{35}$ S]methionine-cysteine $(1 \mathrm{mCi} / \mathrm{ml})$ (PRO-MIX, Amersham). After the pulse, the medium was removed, and the oocytes were washed thoroughly with modified Barths' saline and incubated in the same medium supplemented with excess cold methionine and cysteine. After the medium was harvested, each oocyte was homogenized in $40 \mu \mathrm{l}$ of homogenization buffer (19), supplemented with 50 $\mathrm{mM}$ iodoacetamide. Media and homogenates were frozen in liquid nitrogen and stored at $-80^{\circ} \mathrm{C}$.

26. Homogenates and incubation media were diluted to 1 $\mathrm{ml}$ with Net-gel buffer (14) and incubated with specific antibodies for 4 hours on ice before addition of $50 \mu$ of a $10 \%$ suspension of protein A-Sepharose CL 4B (Pharmacia, Uppsala, Sweden). After overnight incubation at $4^{\circ} \mathrm{C}$, the beads were washed three times with NET-gel buffer and eluted by heating at $95^{\circ} \mathrm{C}$ in SDS-polyacrylamide gel electrophoresis (SDSPAGE) loading buffer [0.062 M tris- $\mathrm{HCl}$ (pH 6.8), 2\% SDS, $10 \%$ glycerol, and $0.001 \%$ bromophenol blue].

27. We thank F. Cozzolino, D. Fesce, I. Haas, A. Helenius, A. Malgaroli, J. Meldolesi, M. Neuberger, A. T. Palamara, R. Pardi, A. Rubartelli, and L. Wrabetz for helpful discussions and suggestions; C. Fagioli for technical help; and S. Trinca for secretarial assistance. This work was supported through grants from the Associazione Italiana per la Ricerca sul Cancro (AIRC), Consiglio Nazionale delle Ricerche (CNR), and Ministero della Sanità (AIDS Special Project) to R.S. S.C. was supported by a fellowship from the Fondazione San Raffaele, in partial fulfillment of a Ph.D. from the Open University, London. A.Ca. is the recipient of a fellowship from AIRC.

19 May 1997; accepted 29 July 1997

\section{Synaptic Efficacy Enhanced by Glial Cells in Vitro}

\section{Frank W. Pfrieger* $†$ and Barbara A. Barres}

In the developing nervous system, glial cells guide axons to their target areas, but it is unknown whether they help neurons to establish functional synaptic connections. The role of glial cells in synapse formation and function was studied in cultures of purified neurons from the rat central nervous system. In glia-free cultures, retinal ganglion cells formed synapses with normal ultrastructure but displayed little spontaneous synaptic activity and high failure rates in evoked synaptic transmission. In cocultures with neuroglia, the frequency and amplitude of spontaneous postsynaptic currents were potentiated by 70 -fold and 5-fold, respectively, and fewer transmission failures occurred. Glial cells increased the action potential-independent quantal release by 12 -fold without affecting neuronal survival. Thus, developing neurons in culture form inefficient synapses that require glial signals to become fully functional.

Brain development and function depends on glial cells, as they guide the migration of neuronal somata and axons (1), promote the survival and differentiation of neurons (2), and insulate and nourish neurons (3, 4). It is not known, however, whether glial cells also promote the formation and function of synapses, although glial processes ensheath most synapses in the brain $(4,5)$. The recent development of methods to purify (6) and culture a specific type of neuron from the central nervous system (CNS) (7) has allowed us to investigate whether CNS neurons can form functional synapses in the absence of glial cells.

We cultured purified postnatal rat retinal ganglion cells (RGCs) without glial cells under serum-free conditions (Fig. 1A)

Department of Neurobiology, Stanford University, School of Medicine, Sherman Fairchild Science Building, 299 Campus Drive, Stanford, CA 94305-5125, USA.

*Present address: Synapse Formation and Function Group, Max-Delbrück-Center for Molecular Medicine, Robert-Rössle-Strasse 10, 13122 Berlin-Buch, Germany. $\dagger$ To whom correspondence should be addressed. E-mail: fpfrieg@mdc-berlin.de
(8) that supported neuronal survival (57 \pm $5 \%$ of neurons survived after 20 days; mean \pm SEM; $n=3$ ), electrical excitability, and the differentiation of axons and dendrites (7). In order to monitor the formation of functional synapses, we recorded spontaneous postsynaptic currents from RGCs (Fig. 1B) (9). After 5 days in culture, $50 \%$ of the RGCs tested (15 of 31 cells) showed low levels of synaptic activity with excitatory postsynaptic currents (EPSCs) occurring at a mean frequency of $3 \pm 1$ $\mathrm{min}^{-1}$ and with a mean peak amplitude of $-11 \pm 1 \mathrm{pA}$ (Fig. 2A). After 20 days of culture, $63 \%$ of the RGCs tested $(n=24)$ displayed spontaneous synaptic activity. The frequency and amplitude of the EPSCs had increased to mean values of $18 \pm 7$ $\min ^{-1}$ and $-16 \pm 1 \mathrm{pA}(n=15$; Fig. 2A), respectively.

To study the effect of neuroglia on synapse formation, we cultured RGCs with glial cells from their target region, the superior colliculus (10). After 5 days of serumfree culture with glial cells, $90 \%$ of the RGCs tested $(n=34)$ showed spontaneous EPSCs. Coculture with collicular glia increased the mean frequency and the mean amplitude of spontaneous EPSCs to $41 \pm$ $12 \mathrm{~min}^{-1}$ and $-29 \pm 3 \mathrm{pA}(n=30)$, respectively (Fig. 2A). After 20 days of coculture with glia, every RGC tested $(n=20)$ showed spontaneous synaptic activity, and the mean EPSC frequency and amplitude were increased to $1265 \pm 212 \mathrm{~min}^{-1}$ and $-78 \pm 8$ pA, respectively $(n=20$; Fig. $2 \mathrm{~A})$. A nearly identical glia-induced enhancement of synaptic activity was observed

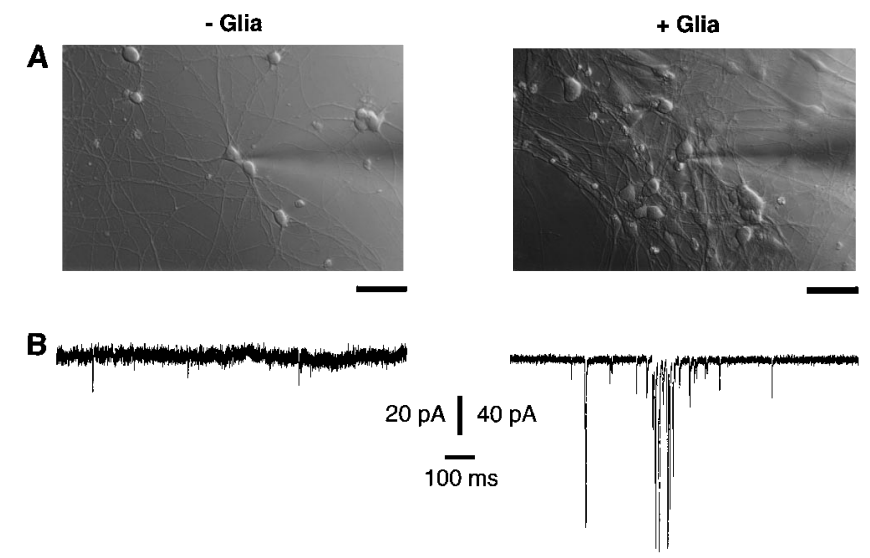

Fig. 1. Spontaneous synaptic activity in purified RGCs that were cultured for 5 days in defined, serum-free medium in the absence (left) or presence (right) of collicular glia. (A) Hoffmann-modulation contrast micrographs of RGCs. Scale bar, $50 \mu \mathrm{m}$. The density of neurons was similar in both cultures. (B) Whole-cell patch-clamp recordings of spontaneous EPSCs from RGCs at a holding potential of $-70 \mathrm{mV}$. 
when RGCs were cocultured with their natural synaptic targets, neurons purified from the superficial layers of the superior colliculus $(11,12)$. Thus, collicular glia strongly enhanced the frequency and amplitude of spontaneous EPSCs in cultured RGCs.

Next, we determined whether glial cells could promote synaptic activity without contacting RGCs. Treatment of RGC cultures with glia-conditioned medium for 10 to 15 days (13) increased the frequency and $425 \pm 84 \mathrm{~min}^{-1}$ and $-31 \pm 2 \mathrm{pA}(n=43)$, respectively, indicating that a soluble signal from glial cells increased synaptic activity. The glial effect was not mimicked by glutrophic factors, or components of the extracellular matrix, because culturing RGCs for 10 to 15 days with these components did not change the level of synaptic activity compared with control cultures (14).

Because our preparation of collicular glia contained a mixture of cell types, we next examined which type of neuroglia increased the synaptic activity by culturing RGCs for 10 to 15 days with purified astrocytes, oligodendrocytes, or microglial cells (15). Both, astrocytes and oligodendrocytes purified from rat optic nerve, increased the frequency and amplitude of spontaneous EPSCs in cultures with (astrocytes, $n=20$ neurons; oligodendrocytes, $n=18$ ) or without (astrocytes, $n=20$; oligodendrocytes, $n=24$, Fig. 2B) direct contact. Collicular microglia did not increase synaptic activity $(n=16)$. Thus, the ability to potentiate synaptic activity was specific to macroglial cells.

Glial cells did not enhance the synaptic activity by improving neuronal survival. the amplitude of spontaneous EPSCs to tamine (8), combinations of various peptide

The percentage of RGCs surviving when cultured above feeding layers of collicular glia or treated with glia-conditioned medium for 10 to 15 days was $107 \pm 5 \%(n=6)$ of the survival rate in glia-free cultures. Furthermore, if glial cells were added to RGCs that were cultured for 10 days without glia, at a time when neuronal survival was stabilized (12), the frequency and amplitude of EPSCs were raised within 3 to 4 days to a similar level $\left(386 \pm 91 \mathrm{~min}^{-1}\right.$ and $-50 \pm 6 \mathrm{pA}, n=24$, two cultures) as in neuron-glia cocultures of comparable age. This confirmed that glial cells increased the synaptic activity independently from neuronal survival.

Glial cells may have affected neuronal excitability, because in cocultures spontaneous EPSCs often occurred in bursts (Fig. $3 \mathrm{~A})$. After 20 days of culture with neuroglia, all neurons tested $(n=20)$ showed bursts of EPSCs (mean frequency $13 \pm 1$ $\left.\min ^{-1}\right)$. These bursts were due to action potential-evoked transmitter release, because action potential-independent release occurs randomly (16). To test whether neuroglia increased synaptic activity by enhancing neuronal excitability, we blocked action potentials with tetrodotoxin (TTX) and recorded miniature EPSCs (mEPSCs) (17). After 12 to 15 days of culture, neuroglia potentiated the mean frequency of mEPSCs by 12 -fold from $8 \pm 1 \mathrm{~min}^{-1}(n=$ 17) in glia-free cultures to $98 \pm 45 \mathrm{~min}^{-1}$ $(n=15)$ and increased their amplitudes significantly $(P<0.001$; KolmogorovSmirnow test; Fig. 3B). The increase in the frequency and amplitude of miniature postsynaptic currents indicated that glial cells acted directly on synapses (18).

Fig. 2. Glial cells potentiated synaptic activity in cultured RGCs. (A) Frequency and mean peak amplitude are shown for spontaneous EPSCs recorded from RGCs that were cultured for 5 (left) or 20 days (right) without ( $\square$ ) and with ( $\square$ ) collicular glia. Each square represents the synaptic activity in each neuron, tested with the EPSC frequency plotted against the mean EPSC amplitude. (B) The effects of different glial cell types on spontaneous synaptic activity are shown. Astrocytes and oligodendrocytes, but not collicular microglia, potentiated the frequency and amplitude of EPSCs (left). The effect did not require direct contact to neurons, as feeding layers of mixed collicular glia, astrocytes, or oligodendrocytes potentiated synaptic activity in RGCs (right). Before the record-
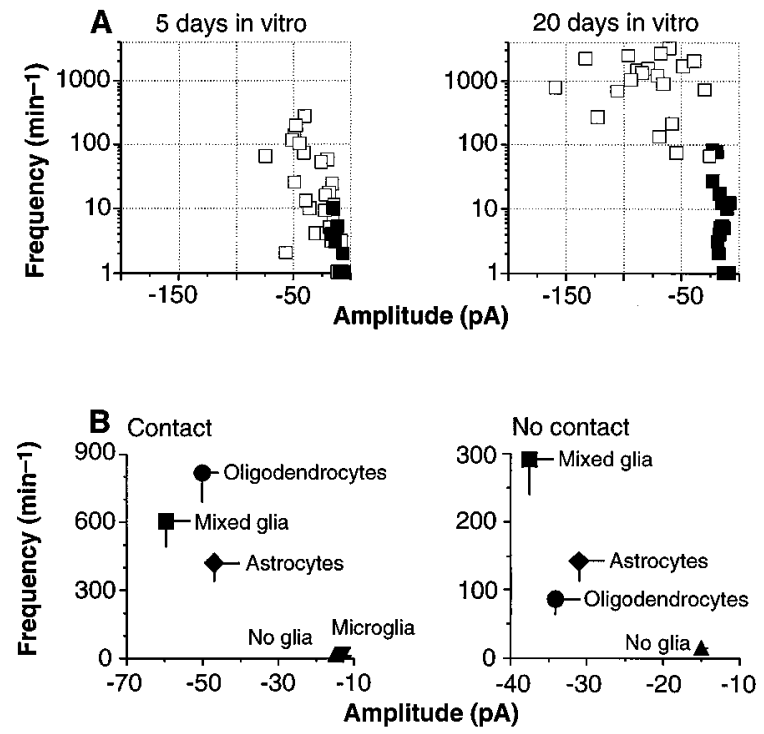

ings, RGCs were cultured for 10 to 15 days with different glial cell types in serum-free medium (15). Averaged EPSC frequencies of all neurons from each culture were plotted against the mean EPSC peak amplitudes. Error bars indicate SEM.
Glial cells can potentiate the frequency of quantal synaptic release by enhancing the number or the efficacy of synapses. In order to test whether RGCs formed synapses in the absence of glial cells, we studied 16- to 21-day-old RGC cultures with the electron microscope and identified synapses on the basis of standard ultrastructural criteria (19). RGCs formed numerous synaptic contacts when cultured without glial cells and, on average, $2.3 \pm$ $0.3(n=3$ cultures $)$ more synapses in the presence of glial cells (20). We observed no apparent differences in the synaptic ultrastructure in glia-free cultures and in cocultures (Fig. 3C). In addition, immunofluorescence staining of glia-free and of cocultures demonstrated that RGCs expressed the synaptic vesicle proteins synapsin I, synaptophysin, and synaptotagmin, as judged by punctate-like staining of
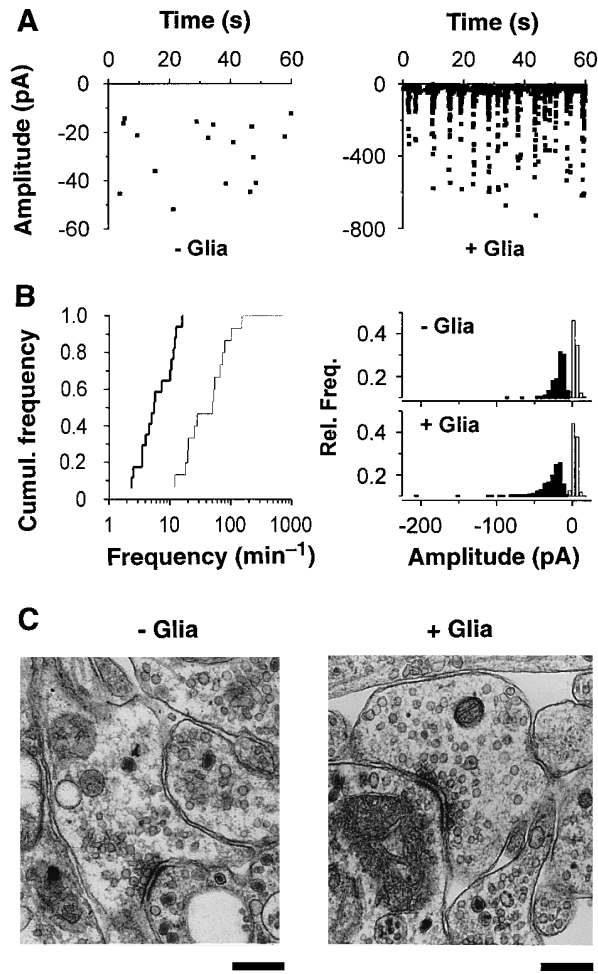

Fig. 3. (A) Bursts of spontaneous synaptic activity occurred frequently in RGCs that were cocultured with glial cells (right) but never in glia-free cultures (left). (B) Glial cells increased the frequency and amplitude of action potential-independent mEPSCs in 12- to 15-day-old cultures (17). Cumulative frequency distribution of mEPSC frequencies (left) in RGC cultures without (thick line) and with (thin line) collicular glia. Amplitude histograms (right) of mEPSCs (filled bars) and recording noise (open bars) in the absence (upper panel) and presence (lower panel) of collicular glia. Cumul. frequency, cumulative frequency; Rel. Freq., relative frequency. (C) Electron micrographs of synapses formed by RGCs that were cultured for 20 days in the absence (left) and presence (right) of glial cells. Scale bars, $0.3 \mu \mathrm{m}$. 

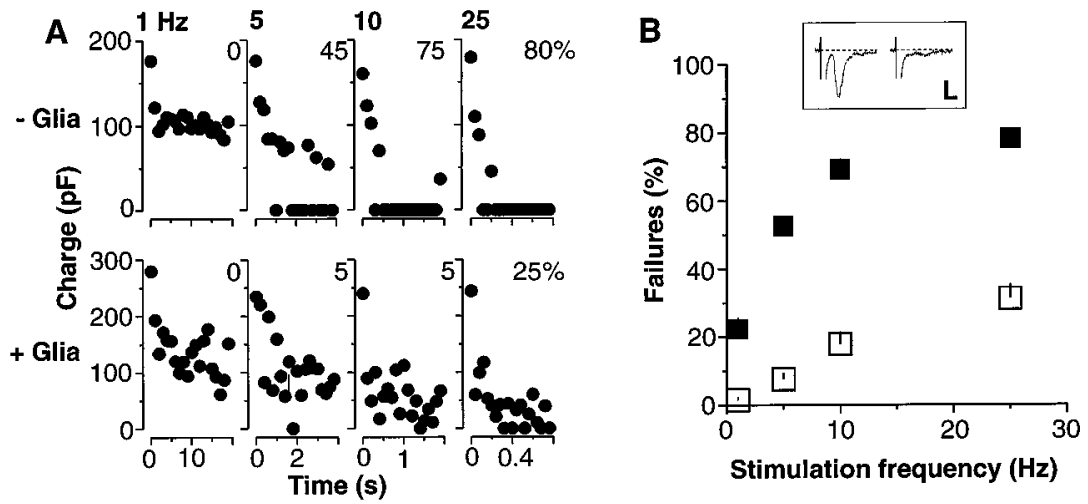

Fig. 4. Glial cells decreased the failure rate of evoked synaptic transmission. (A) In glia-free cultures, the failure rate increased steeply with the stimulation frequency, whereas in the presence of glial cells, synapses could sustain high transmission frequencies. EPSCs were evoked by extracellular stimulation in RGCs cultured for 15 days in the absence (upper panel) or presence (lower panel) of collicular glia. For each RGC, four stimulus trains were applied at the indicated frequencies. The failure rates are indicated. (B) Glial cells decreased the failure rate at every stimulation frequency tested. The failure rates averaged from all neurons tested were plotted against the stimulation frequency. Error bars indicate SEM. RGCs were cultured for 10 to 15 days without $(\mathbf{\square})$ and with $(\square)$ collicular glia. The inset shows an EPSC evoked by extracellular stimulation (left trace) and a stimulation failure (right trace). The membrane potential was held at $-70 \mathrm{mV}$; scale bars indicate $60 \mathrm{pA}$ and $3 \mathrm{~ms}$.

axons (12). Because RGCs formed synaptic contacts in glia-free cultures, the low level of synaptic activity indicated that synapses are inefficient in the absence of glial cells. The twofold increase in the synapse number cannot account for the 12 -fold increase in quantal release, suggesting that glial cells enhanced the synaptic efficacy.

To examine whether glial cells affected synaptic efficacy, we studied evoked synaptic transmission in glia-free and in neuronglia cocultures (21). In 10- to 15-day-old glia-free cultures, extracellular stimulation at $1 \mathrm{~Hz}$ evoked EPSCs in only $22 \%$ of the RGCs tested $(n=94)$. In cocultures with glial cells, every RGC $(n=25)$ showed EPSCs upon extracellular stimulation. In cocultures, the charge transfer of EPSCs evoked at $1 \mathrm{~Hz}$ was, on average, four times larger (mean $189 \pm 42 \mathrm{pF} ; n=25)$ than in glia free cultures $(44 \pm 6 \mathrm{pF} ; n=24)$. Within a stimulus train, some stimuli failed to induce postsynaptic responses (Fig. 4). In glia-free cultures, the percentage of failures at a stimulation frequency of $1 \mathrm{~Hz}$ averaged at $22 \pm 3 \%(n=24$ neurons $)$. When RGCs were cocultured with glial cells, however, the failure rate was reduced to $2 \pm 1 \%(n=$ 25). In both cultures, the failure rate increased with higher stimulation frequencies. In the absence of glial cells, however, this frequency dependence was greater than in the presence of glial cells (Fig. 4A), and at every stimulation frequency tested, the mean failure rates in cocultures were lower than in glia-free cultures (Fig. 4B). If we raised the stimulation frequency from $1 \mathrm{~Hz}$ to $5 \mathrm{~Hz}$ and $10 \mathrm{~Hz}$, the normalized failure rate (21) increased, on average, by $53 \pm 4 \%$ and by $83 \pm 4 \%$, respectively, in glia-free cultures $(n=24)$, but only by $14 \pm 4 \%$ and by $44 \pm 7 \%$, respectively, in cocultures $(n=$ 24). The differences in failure rates at higher stimulation frequencies cannot be explained by the twofold increase in the number of release sites in cocultures (22). In principle, stimulation failures could also be due to unreliable induction or conduction of action potentials. This was unlikely, however, as current-clamp recordings from RGCs (21) in glia-free culture $(n=12)$ showed that antidromic stimulation induced action potentials at every frequency tested. Furthermore, it has been shown previously that failures in synaptic transmission in CNS neurons are caused by the probabilistic nature of transmitter release (23). Thus, the observation of frequent stimulation failures in glia-free cultures indicated that the synapses have low efficacies. The higher reliability of synaptic transmission in cocultures indicated that glial cells enhanced the synaptic efficacy.

In summary, our findings show that the efficient function of developing CNS synapses in vitro depends critically on glial cells, and they raise the question of whether glial cells also regulate synapse function in vivo: Newly formed synapses that are not yet ensheathed by glial cells may be inefficient. Moreover, the efficacy of adult synapses may also depend on their intimate partnership with glial cells (24).

\section{REFERENCES AND NOTES}

1. P. Rakic, Experientia 46, 882 (1990); J. Silver, S. E. Lorenz, D. Wahlsten, J. Coughlin, J. Comp. Neurol. 210, 10 (1982); J. Y. Kuwada, Science 233, 740 (1986).

2. M. R. Kaplan et al., Nature 386, 724 (1997); B. T.
Hosoya, K. Takizawa, K. Nitta, Y. Hotta, Cell 82, 1025 (1995); W. Jones, R. D. Fetter, G. Tear, C. S. Goodman, ibid., p. 1013. For review, see F. W. Pfrieger and B. A. Barres, ibid. 83, 671 (1995)

3. S. R. y Cajal, Histology of the Nervous System of Man and Vertebrates (Oxford Univ. Press, Oxford, 1995); M. Tsacopoulos and P. J. Magistretti, J. Neurosci. 16, 877 (1996).

4. A. Peters, S. L. Palay, H. F. Webster, The Fine Structure of the Nervous System: The Neurons and Supporting Cells (Oxford Univ. Press, Oxford, 1991).

5. S. L. Pomeroy and D. Purves, J. Cell Biol. 107, 1167 (1988).

6. B. A. Barres, B. E. Silverstein, D. P. Corey, L. L. Y. Chun, Neuron 1, 791 (1988).

7. A. Meyer-Franke, M. R. Kaplan, F. W. Pfrieger, B. A. Barres, ibid. 15, 805 (1995).

8. RGCs were purified from postnatal day 8 (P8) Sprague-Dawley rats by immunopanning (6) and cultured at 50 to $100 \mathrm{cells} / \mathrm{mm}^{2}$ on merosin-coated (Gibco) culture dishes (Falcon) in serum-free medium as described (7) containing Neurobasal (Gibco), penicillin (100 U/ml; Gibco), streptomycin (100 mg/ $\mathrm{ml}$; Gibco), insulin (5 $\mu \mathrm{g} / \mathrm{ml}$; Sigma), human brainderived neurotrophic factor (BDNF) $(50 \mathrm{ng} / \mathrm{ml}$; Regeneron), rat ciliary neurotrophic factor (CNTF) (10 $\mathrm{ng} / \mathrm{ml}$; Regeneron), B27 (Gibco), and forskolin (10 $\mu \mathrm{M}$; Sigma). Glutamine, a glutamate precursor that is normally supplied by glial cells, was added to the medium (2 mM; Gibco). The survival of RGCs was determined with the MTT assay (7) at a cell density of 50 to 100 cells $/ \mathrm{mm}^{2}$. All results in this study were obtained from at least three independent culture preparations unless otherwise indicated. Care and handling of Sprague-Dawley rats were in accordance with institutional guidelines.

9. Membrane currents were recorded at room temperature $\left(18^{\circ}\right.$ to $\left.22^{\circ} \mathrm{C}\right)$ and at a holding potential of -70 $\mathrm{mV}$ with the whole-cell configuration of the patchclamp technique as described (7). Postsynaptic currents were analyzed with a computer program (Labview software, National Instruments Corp.). We visually checked whether detected events resembled postsynaptic currents.

10. Collicular glia were prepared as described [K. D. McCarthy and J. De Vellis, J. Cell Biol. 85, 890 (1980)]. Briefly, trypsin-digested and triturated superior colliculi (0.5 mm thick) from P2 rats were cultured in poly-D-lysine-coated tissue culture flasks (Falcon), in a medium that does not support survival of neurons, containing Dulbecco's minimum essential medium, fetal bovine serum (10\%), penicillin $(100 \mathrm{U} / \mathrm{ml})$, streptomycin $(100 \mathrm{mg} / \mathrm{ml})$, glutamine $(2 \mathrm{mM})$, and sodium pyruvate ( $1 \mathrm{mM}$; all supplied by Gibco). After 5 days, the culture flasks were shaken, and the nonadherent cells were washed off. The adherent cells were detached enzymatically and cultured with RGCs at a ratio of 5:1 in serum-free medium (8).

11. To purify collicular neurons, we trypsinized and triturated superficial layers of superior colliculi from P7 rats. Non-neuronal cells were removed from the cell suspension with the Griffonia simplicifolia lectin I (GSL I) isolectin-B4 (microglia, endothelial cells; Vector Labs) and the $\mathrm{O} 4$ antibody (oligodendrocyte lineage). Collicular neurons were selected with a L1specific antibody (ASCS4; Developmental Studies Hybridoma Bank) and cultured for 10 to 15 days at a ratio of $1: 3$ with RGCs in the absence of glial cells. For cocultures of collicular neurons, glia, and RGCs, the collicular cell suspension was plated together with purified RGCs. To record from collicular neurons, we labeled RGCs with Dil $(7 \mu \mathrm{M}$ in Earle's balanced salt solution for $10 \mathrm{~min}$ at $36^{\circ} \mathrm{C}$; Molecular Probes), before they were added to collicular cells.

12. F. W. Pfrieger and B. A. Barres, data not shown.

13. Glia-conditioned medium was obtained from purified collicular glia (10) that were cultured at high density in the same serum-free medium as RGCs (8). The medium was added every second day to RGC cultures at a dilution of $1: 1.5$ together with fresh medium.

14. We list the combinations of peptide growth factors tested with the mean EPSC frequency measured in each culture: (i) base morphogenetic protein-2 (BMP-2)/BMP-4/BMP-7/interleukin (IL-3)/IL-6/IL-7/ basic fibroblast growth factor: $22 \pm 10 \mathrm{~min}^{-1}(n=11$ 
neurons), (ii) hepatocyte growth factor/nerve growth factor/transforming growth factor- $\beta$ (TGF $\beta$ )/glial cel line-derived neurotrophic factor: $36 \pm 13 \mathrm{~min}^{-1}(n=$ 12), (iii) TGF $\alpha$ /glial growth factor: $8 \pm 5 \mathrm{~min}^{-1}(n=8)$, and (iv) neurotrophin-3/plate-derived growth factorAA: $16 \pm 6 \mathrm{~min}^{-1}(n=12)$. Each of these factors promoted cell survival, proliferation, or differentiation in other assays performed in our lab. To test the effect of extracellular matrix components, we cultured RGCs on Matrigel (Collaborative Biomedica Products) (mean EPSC frequency of $21 \pm 11 \mathrm{~min}^{-1}$, $n=7$ ) or in serum-free medium containing chondroitin sulfate proteoglycan (5 $\mathrm{g} / \mathrm{ml}$; Collaborative Biomedical Products) (mean frequency $2 \pm 2 \mathrm{~min}^{-1}$ $n=6)$. Both components were active, as they fasciculated neurites of RGCs.

15. Astrocytes (7) and oligodendrocytes [B. A. Barres et al., Cell 70, 31 (1992)] were purified by immunopanning from postnatal rat optic nerves as described, because these glial cell types cannot be purified from other brain areas. Microglial cells were purified from the superior colliculus of $\mathrm{P} 8$ rats by immunopanning with a goat anti-rat immunoglobulin $G$ antibody (Jackson ImmunoResearch Laboratories) and a rat anti-mouse Thy 1.2 antibody (Boehringer). RGCs were cultured with each glial cell type at a ratio of three to five glial cells per neuron in the defined serum-free medium (8). In order to avoid contact between neurons and glial cells, we cultured RGCs on cover slips above glial feeding layers.

16. P. Fatt and B. Katz, J. Physiol. (London) 117, 109 (1952).

17. Miniature EPSCs were isolated by adding TTX (10 $\mu \mathrm{M}$, Sigma), bicuculline methylchloride (50 $\mu \mathrm{M}, \mathrm{RBI})$ and D-2-amino-5-phosphonopentanoic acid (50 $\mu \mathrm{M}, \mathrm{RBI})$ to the extracellular recording solution.

18. The average whole-cell resting membrane potentia in RGCs cultured for 10 to 15 days without $(-48 \pm 1$ $\mathrm{mV}, n=22)$ or with glial cells $(-49 \pm 1 \mathrm{mV}, n=19)$ was not different. This, however, does not rule out that glial cells increased quantal release by depolarizing nerve terminals.

19. For electron microscope analysis, cultures were fixed with glutaraldehyde $(2.5 \%$ in phosphate-buffered saline for $30 \mathrm{~min})$, treated with osmium tetroxide (1\% for $30 \mathrm{~min}$ ), dehydrated, and embedded in Epon 812 (Polysciences). For each culture, ultrathin sections were cut from at least two blocks with a glass knife, stained with lead citrate and uranyl acetate, and examined with a Phillips EM 410. Ultrastructura criteria to identify synapses were a pre- and a postsynaptic density, a synaptic cleft, and more than two synaptic vesicles in the presynaptic active zone. We counted synapses in photomicrographs ( $\times 18,500$ magnification) from randomly chosen ar eas in one to seven sections per block. The number of neurons in glia-free cultures was estimated by counting neuronal cell bodies in the sections. Because the density of neurons was similar in glia-free and in cocultures, we could directly compare the number of synapses in equal numbers of micrographs from each culture condition.

20. In three glia-free cultures, we counted 168, 104, and 43 synapses in 109,60 , and 61 micrographs with approximately 20,29 , and 8 neurons. In the corresponding cocultures with glia, we found 373,179 , and 87 synapses in 104, 57, and 44 micrographs, respectively. Thus, glial cells increased the number of synapses per micrograph by $2.3,1.8$, and 2.8 , respectively.

21. EPSCs were evoked by extracellular stimulation as described [F. W. Pfrieger, K. Gottmann, H. D. Lux, Neuron 12, 97 (1994)]. Briefly, a glass pipette (tip diameter: 1 $\mu \mathrm{m})$, filled with extracellular solution was placed near neurite, where brief $(100 \mu \mathrm{s})$ current pulses $(0.2 \mathrm{~mA})$ evoked EPSCs in the postsynaptic neuron clamped at a holding potential of $-70 \mathrm{mV}$. At each stimulation site, two trains of 20 stimuli were applied at 1, 5, 10, and 25 $\mathrm{Hz}$, respectively. Action potentials in the postsynaptic neuron were blocked by including the lidocaine derivate QX-314 (10 mM, RBI) in the intracellular recording solution. For the analysis of EPSCs, membrane currents were integrated over a time window of $8 \mathrm{~ms}$ beginning at the EPSC onset. For each stimulus, the presence of an EPSC or a stimulation failure was determined by the experimenter. In order to normalize failure rates at 5 and $10 \mathrm{~Hz}$, for each RGC we subtracted the failure percentage at $1 \mathrm{~Hz}$ and divided by the rate at $25 \mathrm{~Hz}$. To test the reliability of extracellular stimulation, we performed current-clamp recordings with 6,7-dinitroquinoxaline-2,3dione $(10 \mu \mathrm{M})$ added extracellularly to exclude actionpotential induction by synaptic currents.

22. The binomial model of transmitter release [see E. M. McLachlan, in International Review of Physiology: Neurophysiology III, Volume 17, R. Porter, Ed. (University Park Press, Baltimore, MD, 1978), pp. 49117] was used to estimate whether the different failure rates in glia-free cultures and in cocultures can be explained by the difference in the number of synapses. According to the binomial equation, the number of trials without release $\left(x_{0}\right)$ in a series of $N$ trials is given by $x_{0}=N \cdot q^{n}$, and the failure rate is $f=x_{0} / N=q^{n}$, with $q$ denoting the mean probability of no release $(q=1-p)$ and $n$ the number of release sites. Assuming that glial cells do not change the release probability $\left(q_{+ \text {glia }}=q_{\text {-glia }}\right)$, we can calculate the increase in the number of release sites that would cause the observed changes in the failure rates ac- cording to $n_{+ \text {lia }} / n_{\text {- }}=\log \left(f_{+ \text {lia }}\right) / \log \left(f_{-g \text { ia }}\right)$. The values for $n_{+ \text {glia }} / n_{\text {-glia }}$ are 2.8 at $1 \mathrm{~Hz}, 4.0$ at $5 \mathrm{~Hz}$ and 4.8 at 10 and $25 \mathrm{~Hz}$. The ratios at higher frequencies exceeded the observed twofold increase in the synapse number, indicating that glial cells enhanced the efficacy of individual release sites.

23. S. Redman, Physiol. Rev. 70, 165 (1990); C. Allen and C. F. Stevens, Proc. Natl. Acad. Sci. U.S.A. 91 10380 (1994); P. J. Mackenzie, M. Umemiya, T. H. Murphy, Neuron 16, 783 (1996).

24. F. W. Pfrieger and B. A. Barres, Curr. Opin. Neurobiol. 6, 615 (1996)

25. Supported by the Human Frontier Science Program Organization and the Deutsche Forschungsgemeinschaft (F.W.P.) and by The Searle Scholar Program/ The Chicago Community Trust (B.A.B.). We thank T. L. Schwarz and R. W. Tsien for valuable comments on the manuscript, S. Shen for ultrathin sections, F. Thomas for kind help with the electron microscope, and R. Scheller for generously providing an anti-synaptotagmin antibody. We also thank Regeneron for the kind gift of recombinant BDNF and CNTF.

3 March 1997; accepted 23 July 1997

\section{“Killer" Impacts and Life’s Origins}

The recent report by Christopher P. McKay and William J. Borucki (1) brought the consequences of impact shocks for the origin of life on Earth into focus anew. One could, however, imagine further positive consequences of large and small impacts for the prebiotic environment.

If one accepts the "RNA-world" scenario (2) for the origin of life on Earth, one has to assume a massive production of activated ribonucleotides or RNA-oligonucleotides. Whereas nucleotide bases and sugars could be produced from hydrogen cyanide (HCN) and formaldehyde - for which plausible prebiotic synthesis-mechanisms in the early Earth atmosphere exist (3) - the triphosphate part of the ribonucleotide would have to be mobilized from the lithosphere and brought into contact with the sugars and bases. Furthermore, a ready source of free enthalpy would be required to drive the cycles of polymerization (reproduction) and destruction (selection) of RNA chains until ribozymes (or even enzymes) arose that could couple the self-replicating RNA system to other, not so easily available sources of free energy, like, for example, solar radiation. Several researchers have proposed polyphosphate as this primary source of free enthalpy [see references in (4)].

In this respect, the paper by Yamagata et al. (5) about polyphosphate synthesis by heating of phosphate rocks with water vapor of over $1000^{\circ} \mathrm{C}$ and subsequent rapid cooling is most instructive. But instead of a synthesis route consisting of production of $\mathrm{HCN}$ and formaldehyde by lightning or ultraviolet radiation followed by rain out and subsequent reaction with polyphos- phates produced in volcanoes (5), a second, maybe more efficient, way of ribonucleotide synthesis should be examined, which is also in line with current thought about the accretion of the Earth by planetesimals and its continuing bombardment even after the oceans and atmosphere had formed.

Fegley et al. (6) demonstrated the synthesis of HCN by meteoric impact on Earth's early atmosphere, while Sleep et al. (7) described the consequences of large impacts for the prebiotic enviroment. Large impacts could have repeatedly vaporized not only the entire ocean of the early Earth, but also enough rock to create 100 bar of rock vapor and suspended droplets with a temperature of $2000^{\circ} \mathrm{C}(7)$. Smaller impacts that vaporize only the photic zone of the oceans were also discussed by Sleep et al. (7). It would be interesting to examine whether, under these conditions, (poly-)phosphates would be produced and in what quantities. Probably no activated polyphosphate would survive the cooling time after a so-called "ocean blaster" had vaporized the whole ocean, but even after somewhat smaller impacts the resulting rock vapor probably produced polyphosphates like the heated phosphate rock/basalt mixure used by Yamagata et al. (5). As the extraction efficiency rises with increasing temperature (5), the higher temperatures should compensate for the fact that natural occurring rocks contain over one order of magnitude less phosphate than the model substances (4). The ocean would boil under the influence of the infrared radiation of the rock vapor, but the ocean depths would remain cool (7) and could act as a cold trap as in Yamagata's experiment. 